\title{
Review
}

\section{Research progress on influencing factors of hospital infection and prevention and control measures}

Wenlong He, Lingbo Meng, Yaogang Wang

TianJin Medical Univercity School Of Public Health, TianJin, China

\section{Keywords \\ Hospital infection; Influencing factors; \\ Prevention and control}

\section{Correspondence}

Yaogang Wang, E-mail: hwltme_edu@163.

com

DOI: 10.1515/ii-2017-0101

\begin{abstract}
Hospital infections are associated with the emergence of hospitals. As the understanding of hospital infections deepen and prevention and control measures improve, hospital infections have become manageable. In recent years, affected by the increase in invasive treatment technology, antimicrobial abuse, and other factors, the control of hospital infection has encountered new problems. This paper reviews the influencing factors of hospital infections and their prevention and control measures.
\end{abstract}

Hospital infections are also known as nosocomial or hospital-acquired infections (hospital-associated, hospitalacquired, hospital, and nosocomial infections). The "Diagnostic Criteria of Nosocomial Infection (Trial)" issued by the Ministry of Health in 2001 defined hospital infections as infections acquired by hospitalized patients within the hospital, including infections during hospitalization and those acquired in the hospital but found after patient discharge; they do not include the infections that already exist before or upon hospital admission. The infections obtained by hospital staff inside hospitals are included ${ }^{[1]}$.

Hospital infections have presented since the advent of medical care. As modern medical science develops, the understanding of hospital infection gradually deepens; however, currently, hospital infection remains an important threat to patient safety. In 2005, a cross-sectional survey by the World Health Organization showed that the number of hospital-infected patients worldwide to exceed 14 million $^{[2]}$. TheU.S. Centers for Disease Control and Prevention estimates that nearly 1.7 million people are infected in hospitals in the United States annually, and approximately 9.9 million of which die due to the merger of various bacterial infections ${ }^{[3]}$. China's $15^{\text {th }}$ National Hospital Infection Management Academic Annual Meeting revealed that among the tens of millions of hospitalized patients each year, approximately $10 \%$ encounter hospital infections that come with an additional expenditure from medical expenses of up to billions of dollars, thereby bringing a grave threat to patients 'life and health and posing major direct and indirect economic burdens.
The United States began to study hospital infection in the 1950s, and, in the 1970s, had set up a national hospital infection monitoring network and brought the hospital infection rate under control to approximately $5 \%{ }^{[4]}$. China's hospital infection management work started late. The study of hospital infections did not start until the 1980s.In 1986, the Medical Department of the Ministry of Healthset up a hospital infection monitoring and coordination group and established national hospital infection monitoring network ${ }^{[5]}$, which consisted of hospitals in 30 provinces, municipalities, and autonomous regions. The data analysis by Wen et al. ${ }^{[6]}$ of the hospital infection cross-sectional survey reported by the 2010 Ministry of Health Hospital Infection Monitoring Network show that the prevalence of hospital infection in China is only $3.60 \%$; however, hospital infection outbreaks occur occasionally in hospitals around China. Chen Ping et al. ${ }^{[7]}$ retrospectively analyzed the hospital infection outbreaks in China from January 1980 to December 2009, and the results showed that medical staff hand cross-infection, blood product contamination, lack of disinfection and quarantine measures, and operating procedure violations are the main causes of outbreaks. China still has a long way to go in preventing and controlling hospital infections.

\section{Influencing factors of hospital infections}

Hospital infection management has not been given enough

The incidences of hospital infections are related with tangible 
conditions and the functional layout of wards, operating rooms, clinics, supply rooms, and other departments. A survey by Chen Yue et al ${ }^{[8]}$ in 2011 showed that equipment aging, lack of equipment, and uncompleted reconstruction of functional parts exist in basic medical units. Management of medical units with improved conditions often only focuses on tangible infrastructure construction and ignores the "soft needs" of hospital infection management, resulting in unreasonable allocation of medical resources and equipment and the prevalence and frequent occurrence, and even the popularity, of hospital infections.

\section{Hospital infection-related knowledge, attitudes, and behavior of medical staff}

The mastery of hospital infection-related knowledge of the medical staffis directly related to the prevention and control of hospital infections. The course contents in medical colleges seldom involve hospital infection-related knowledge; therefore, majority of the medical staff has little knowledge on hospital infections and lack of awareness of prevention and control ${ }^{[9]}$. For example, not strictly abiding by operating procedures, diagnoses and treatments, and hand cleaning and disinfection before and after nursing the patients will all increase the risk of hospital infections.

\section{Not enough attention paid to hand hygiene}

In diagnosing, treating, and nursing, the hands of medical staff are susceptible to pathogens and thus become crucial media. The bacteria on the hands of medical staff are grouped into temporary and permanent bacteria. Temporary bacteria are pathogens that are related to hospital infection. Such pathogens can be obtained from infected wounds, drainage openings, and patients' secretions as well as normal, intact skin areas. At present, hand hygiene compliance of medical staff remains low. Shen et al. ${ }^{[10]}$ surveyed the hand hygiene compliance of medical staff in Shanghai Grade 2Aand above general hospitals, and the results showed that hand hygiene compliance is only $16.8 \%-62.3 \%$.

\section{Increase in invasive operations}

In recent years, as medical technology developed and new devices and technologies are introduced, invasive treatment measures, including hem dialysis, indwelling catheter, endoscopy, arterio venous catheter, and organ transplantation, increased significantly and so has the opportunity of pathogenic microorganisms to invade the human body ${ }^{[11]}$. A survey by the national hospital infection monitoring network of patients who receive continuous hem dialysis showed that the hepatitis $\mathrm{C}$ virus infection rate of hospitals with large workloads is high in patients who receive long-term dialysis and those who have received dialysis in multiple hospitals ${ }^{[12]}$. Non-proficient or substandard operation easily leads to hospital infections. The investigation by Wu Xiao et al. ${ }^{[13]}$ on the quality of endoscopy disinfection in Grade 2 hospitals in Jiangsu Province showed that as daily consultation increased, due to lack of time, the operating staff often do not follow the prescribed procedures for endoscopy cleaning and disinfection, which may proliferate hospital infections.

\section{Misuse of antibiotics, hormones, and other drugs}

Antibiotics, which are widely used in clinical practice to prevent and treat various infectious diseases, are important in reducing hospital infections. However, antibiotic misuse or abuse can cause the imbalance of bacteria spectra in the body and increase drug-resistant strains, thereby increasing the chance of infection in patients ${ }^{[14]}$. Chemotherapy, radiotherapy, immunosuppressive agents, and hormone therapy can inhibit the immune system, thereby increasing the risk of infection in patients.

\section{Prevention and Control of Hospital Infection}

Hospital infections have three basic conditions (or three links), that is, the source of infection, route of transmission, and susceptible host. Infection prevention and control is to intervene between the three links, that is,control and eliminate the infection source, cut off theroute of transmission, and protect the susceptible host. Moreover, regulationsin hospital infection management organizations, related functional departments, and new concepts of evidence-based medicine developed in the 1990s have played important roles in infection prevention and control.

Establish a hospital infection management organization and define the roles of hospital infection management positions

Medical institutions at all levelsshould establish a hospital infection management responsibility system, formulate and implement the hospital infection management rules and regulations,strictly enforce the relevant operational norms and work standards,effectively prevent and control hospital infection, and prevent the transmission of 
infectious pathogens, drug-resistant bacteria, opportunistic pathogens, and other pathogenic microorganisms ${ }^{[15]}$.

\section{Strengthen the training for medical staff to improve hospital infection awareness, prevention, and control \\ Enforcing the hospital infection training for medical staff in basic medical units is crucial and should be among the important components of hospital infection management ${ }^{[16]}$. Hospital infection management professionals should have expertise in hospital infection prevention and control and be able to undertake hospital infection management and technical operation ${ }^{[15]}$.}

\section{Strengthen disinfection, sterilization, and quarantine measures}

Disinfection and sterilization are important measures to prevent hospital infection. Medical staff should pay attention to disinfection and sterilization, adhere to these concepts, strictly abide by the relevant provisions on disinfection and sterilization in the "Disinfection Management Regulations," strictly implement the "Technical Specifications of Quarantine, "and based on route of transmission ofpathogens, take appropriate quarantine measures ${ }^{[17]}$.

\section{Raise awareness of hand hygiene and improve hand washing facilities}

Hand hygiene is one of the most important measures to control hospital infections ${ }^{[18]}$ as well as the most economical and effective ${ }^{[19]}$. Numerous epidemiological studies confirmed that the hand is an important route of spreading hospital infections. Proper handwashing can significantly reduce potential pathogens carried by hands and effectively eliminate transmission by contact. Foot or inductive switchesare the best choice for handwashing facilities. Hospitals that cannot afford these facilities can choose elbow control switches. Moreover, given that solid soap is easily contaminated, liquid soap and hand sanitizer may be used. Furthermore, the laboratory, dentistry unit, ICU, transfusion room, and other departments should be equipped with fast hand sanitizers to facilitate hand disinfection.

\section{Regulate and reduce invasive operations}

Invasive operations are common hazards for hospital infection; therefore, during invasive operations, the staff should strictly follow disinfection, quarantine, and aseptic technologies to prevent human-induced pathogenic microorganisms from invading the body. The incidence of hospital infections increase with the number of patients receiving invasive operations ${ }^{[11]}$. Therefore, unnecessary invasive procedures should be minimized to reduce the risk of hospital infection.

\section{Rational use of antibiotics; strengthen the monitoring of drug-resistant bacteria}

Antibiotics should be selected reasonably based on the patient's condition. Moreover, the infection management of multi-drug resistant bacteria should be strengthened, the principles of antibiotics use should be mastered, antibiotics should beselectedscientifically based on drug susceptibility test results ${ }^{[20]}$, and the use of preventive antibiotics should be strictly controlled. Concurrently, the monitoring of strong drug-resistant bacteria should be strengthened, and the bacterial resistance spectrum should be grasped timely to guide the rational use of clinical drugs ${ }^{[21]}$.

\section{Strengthen the hospital infection and disinfection quality monitoring}

In 1986, the Medical Department of the Ministry of Health set up a national hospital infection monitoring system. After a long period of comprehensive monitoring, it already has a basic grasp of the overall incidence of hospital infections in China, the incidence of hospital infections in specialized hospitals, the use of antibiotics, common pathogens of hospital infection, and the drug resistance spectrum that provides a scientific basis for the control of hospital infection $^{[22]}$.

Strengthening the quality of disinfection monitoring and timely detection of hospital infection risk are important in hospital infection prevention ${ }^{[23,24]}$. Disease prevention and control institutions at all levels should have a continuous and systematic plan for hospital disinfection quality monitoring, thereby providing scientific data for health administrative departments to develop systems, make decisions, and guide medical institutions in preventing and controlling hospital infections.

Enforce hospital infection management based on the concept of evidence-based medicine

Evidence-based medicine is a new discipline developed in the beginning of the 1990s, and its core idea is that "any medical and health program and decision-making step should follow the objective evidence of clinical research to produce the best evidence." The development of evidencebased medicine provided a new model for hospital infection 
control $^{[25]}$. For example, in recent years, the incidence of surgical site infection has become significantly lower than that of preoperative hair removal without affecting the surgical field of view. If you have to remove hair, scissors or depilatory agents are better choices ${ }^{[26]}$. This discovery subverts the past hair removal methods. Therefore, all medical practice must rely on the results of scientific research. Hospital infection control is highly professional; the staff needs to have knowledge on clinical medicine, microbiology, pharmacy, epidemiology, and other aspects; and in practical work, they should keep pace with the times, constantly update their knowledge, and apply the results of evaluation to the hospital infection management process, thereby effectively protecting the lives of patients and the safety and health of the medical staff.

\section{Declarations}

\section{Acknowledgements}

This work was partially supported by National Natural Science Foundation of China (No.71473175).

\section{Competing interests}

The authors declare that they have no competing interest.

\section{Authors' contributions}

WL He and LB Meng made the literature analysis and wrote, discussed and revised the manuscript of this review. YG Wang critically analyzed and corrected the manuscript. All authors read and approved the final manuscript.

\section{References}

1 People's Republic of China Ministry of Health. Diagnostic Criteria of Nosocomial Infection (Trial). Zhonghua Yi Xue Za Zhi, 2001, 81(5): 314-320.

2 Shen Z. Overview of Global Hospital Infection Control [C] / / Chinese Preventive Medicine Association. Blue Book of Preventive Medicine Discipline Development (2006 volume). Beijing: Chinese Preventive Medicine Association, 2006: 101-107.

3 Pollack A. Rising threat of infections unfazed by antibiotics $[N]$. The New York Times, 2010-02-27 (26).

4 Graves N. Economics and preventing hospital-taken infection. Emerg Infect Dis, 2004,10 (4): 561-566.

5 Liu G, Cao S, Wen J. Prevention and control of hospital infection. Zhongguo Gao Deng Yi Xue Jiao Yu, 2007, (9): 95.

6 Wen X, Ren N, Wu A. 2010 cross-sectional survey of national hospital infection: infectious pathogen distribution and its drug resistance.
Zhongguo Gan Ran Kong Zhi Za Zhi, 2012, 11(1): 1-6.

7 Chen P, Liu D. Epidemiological characteristics and countermeasures of China's nosocomial infection outbreak in the recent 30 years. Zhongguo Gan Ran Kong Zhi Za Zhi, 2010, 9(6): 387-392, 399.

8 Chen Y, Tan Z, Sun W, et al. Disinfection status of basic health service agencies in Jiangsu Province. Jiangsu Wei Sheng Bao Jian, 2012,14(2): 23-24.

9 Ye Y, Huang K. Application of Hospital infection management process in dentist clinics. Zhongguo Wu Zhen Xue Za Zhi, 2010, 10(25): 6167-6168.

10 Shen Y, Hu B, Zhou Q et al. Survey of hand hygiene compliance in 66 hospitals in Shanghai. Zhonghua Yi Xue Gan Ran Xue Za Zhi, 2012, 22(12): 2585-2587.

11 Zhang $\mathrm{Q}$ Zhang $\mathrm{H}$. Analysis of current situation and countermeasures of hospital infection caused by invasive operations. Xi Bu Yi Xue, 2010, 22(8): 1568-1569.

12 Ren N, Wen X, Wu A. Analysis of current situation by national Hospital Infection Monitoring Network of patients with persistent hemodialysis hepatitis C virus infection. Zhongguo Gan Ran Kong Zhi Za Zhi, 2011, 10(6): 412-415

13 Wu X, Chen W, Xu Y, et al. Analysis of disinfection of endoscopy in Grade 2 hospitals in Jiangsu Province. Zhonghua Yi Xue Gan Ran Xue Za Zhi, 2010, 20(23): 3724-3725.

14 Wang $X$. Hospital infection management difficulties and countermeasures. Shanghai Yu Fang Yi Xue, 2007, 19(3): 141-142.

15 Wu A, Li C. Hospital infection management. Zhongguo Zhi Ye Yao Shi, 2012, 9(6): 42-48.

16 Wang F, Fan Q. Training evaluation of disinfection and sterilization and hospital infection. Xian Dai Yu Fang Yi Xue, 2010, 37(11): 2056-2057.

17 Yao R, Cao S. Modern hospital infection management and control. Dang Dai Yi Xue, 2010, 16(2): 41-42.

$18 \mathrm{Gu}$ X. Research progress of medical staff hand hygiene. Zhonghua Yi Xue Gan Ran Xue Za Zhi, 2012, 22(8): 1757-1760.

19 Bilski B, Kosiński B. An analysis of selected hands hygiene habits in selected population of nursing staff. Med Pr, 2007, 58 (4): 291-297.

20 Liu Y, Zou J, Hao X, et al. Monitoring and drug resistance of nosocomial infection pathogens in ICUs. Zhongguo Zong He Lin Chuang, 2012, 28(12): 1293-1295.

21 Zhou W, Chen Y, Li G, et al. Study on drug resistance of major pathogens in Chengdu No. 363 hospital from 2009 to 2011. Xian Dai Yu Fang Yi Xue, 2012, 39(20): 5375-5379.

22 Ding Y, Zhang W, Yin X, et al. Comparative analysis of infection monitoring system in national hospitals of China, USA and Germany. Yi Xue Yu Zhe Xue, 2007, 28(8): 50-51.

23 Zhu L, Gao L, Cao P, et al. Status and problems of disinfection in China' s medical institutions. Zhongguo Xiao Du Xue Za Zhi, 2010, 27(6): 720-723.

$24 \mathrm{Xu} \mathrm{Y,} \mathrm{Chen} \mathrm{W,} \mathrm{Tan} \mathrm{Z,} \mathrm{et} \mathrm{al.} \mathrm{Monitoring} \mathrm{results} \mathrm{report} \mathrm{of} \mathrm{disinfection}$ quality in two large general hospitals. Zhongguo Xiao Du Xue Za Zhi, 2012, 29(2): 117-119. 
25 Wu X, Sun B, Gong H. Establishment of hospital infection control infection. Cochrane Database Syst Rev, 2011, (11): CD004122. process and the application of evidence-based medicine. Zhonghua Yi Xue Gan Ran Xue Za Zhi, 2008, 18(6): 805-808.

26 Tanner J, Norrie P, Melen K. Preoperative hair removal to surgical tissue 